\title{
Full Recovery of the Patient With Bell's Palsy Within Two to Six Weeks After Single Course of Pulsed Electromagnetic Therapy-Case Reports
}

\author{
Lauren Gorelick $^{1 *}$, Ayala Rozano-Gorelick ${ }^{2}$ \\ ${ }^{1}$ Pain Clinic, Carmiel, Israel \\ ${ }^{2}$ Cllalit Health Services- Bar Ilan medicine faculty, Zefat, Israel
}

*Corresponding Author: Lauren Gorelick, Pain Clinic, Kfar Rosh Haniqra, POB 91, 2282500, Israel, E-mail: gorelick@netvision.net.il

Received: 12 May 2018; Accepted: 18 May 2018; Published: 21 May 2018

\begin{abstract}
Bell's palsy (BP) is the one of the most common causes of facial nerve neuropathy cause facial paralysis. Etiology of the BP is not known. Diagnosis made according to anamnestic data and clinical examination. Other pathology as CVA, Lyme disease, viral infection, brain tumors must be excluded. A degree of nerve damage graduate according to modified House-Brackmann score. Corticosteroids, antiviral therapy, symptomatic treatment, physiotherapy, alternative medicine and surgical intervention, in some severe cases with residual palsy, are the treatments of choice. About $70 \%$ of patients had a full recovery and in some cases without any treatment. However, $30 \%$ had residual damage to the facial nerve, and functional disability and $12 \%$ have a recurrence. In the article, authors present new treatment modality known as Pulsed Signal Therapy (PST ${ }^{\circledR}$ ) that was applied as management for severe pain around the ear of the affected side and surprisingly cased for full recovery of two patients with BP between two to six weeks after treatment.
\end{abstract}

Keywords: Bell’s palsy; Electromagnetic fields; Pulsed Signal Therapy

\section{Introduction}

Bell's palsy (BP) is one of the most common causes of a facial nerve paralysis that is not related to stroke. Etiology of the disease not clearly known. Acute onset of the unilateral facial nerve paresis or paralyzes case the functional, cosmetic and mental disability of the patients. In most cases, clinical presentation is self-limited or resolved after corticosteroid and symptomatic treatment. However, the facial paresis or paralysis may cause significant temporary 
oral incompetence and an inability to close the eyelid, leading to potential eye injury [1]. Patients graduate according modified House-Brackmann score. Standard corticosteroid therapy of BP is recommended by guidelines in combined with other symptomatic treatments if needed. However, diabetes, pregnancy, hypertension and intolerance to corticosteroids may be contraindications to steroid therapy. No other medical modalities are known as useful for treatment of BP. About 70\% of patients had full recovery and in some cases without any treatment. However, 30\% had residual damage of facial nerve and functional disability and $12 \%$ have a recurrence [2]. In the current article, authors describe two patients with BP who had been referred to a pain clinic due to severe pain around the ear of the affected side when all treatments failed.

In the last 20 years, electromagnetic fields are widely used in neurology, psychiatry, rheumatology, orthopedics and dermatology, both in diagnosis and in therapy [3]. Magnetotherapy provides a noninvasive, safe, and comfortable method to directly treat the site of injury, the source of pain and inflammation, and a variety of diseases and pathologies. Pulsed electromagnetic fields are one the important modality in magnetotherapy [4]. Pulsed Signal Therapy (PST®) is the innovative method of treatment uses a computerized control device that generates and releases by pulsed DC quasi-rectangular waves 1-30 Hz frequency modulated impulse (six frequency sources). Field intensity is no greater than 20 Gauss. PST ${ }^{\circledR}$ is an effective treatment alternative and requires in most cases only one course of treatment to provide sustained pain relief and restoration of normal function. This device acts as the external biophysical signal of physiological energy parameters and waveform. It passively induces 'fluid flow', creates 'streaming potentials', to trigger biochemical coupling, signal transduction and elicitation of the repair and regenerative processes [5]. Pulsed electromagnetic fields as noninvasive, painless and non-pharmacological treatment may be applicable in the cases when conventional medicine is non-effective or contraindicated and no other modalities for the treatment.

In this article authors describe two case reports of the BP when the patients started PST ${ }^{\circledR}$ as the symptomatic pain treatment and ended it with full recovery of the disease in two to six weeks after acute onset of the disease.

\section{Case Report}

In the first case, a 58-year-old female presented to the pain clinic with left side Bell's palsy (BP), that onsets acutely five days early without any history of trauma or infectious disease. She examined by the neurologist in the emergency room of the local hospital and outpatient neurologic clinic three days after onset of the disease symptoms. The diagnosis of the BP was made according to history and physical examination. According to the data in the patient medical chart, complete paralysis of the muscle left side of the face accompanied by ipsilateral severe pain around the ear, significant drooping of eyelid and corner of mouth on the left side, Bell phenomenon, excessive tearing of the left eye and some impaired of taste was the symptoms at the time of clinical examination in emergency room. Oral Prednisolone $50 \mathrm{mg}$. daily and symptomatic treatment of eye and oral symptoms started immediately as the diagnosis made. On the second day of the Prednisolone treatment side effects of hormonal therapy appeared in the patient. She consulted with the neurologist that stope the Prednisolone. However, no any 
other treatment was recommended except symptomatic. The patient presented to the pain clinic with left side complete BP accompanied by pain around the left ear, which not responded to analgesics and NSAID. The patient was graduated as severe according to modified House-Brackmann score. After explanations about pain clinic options for pain management in the clinic, the patient chose optional treatment by PST®. The patient oral and written agreement were obtained. The standard protocol of nine days one-hour daily treatment of PST® started immediately on the home-based device of the PST®. After fifth-day of the procedures recorded significant improvement in pain and withdrawal of clinical signs of disease. The patient completed nine consecutive treatments and fool recovery achieved within two weeks after acute onset of BP.

In the second case, 57-year-old previously healthy male, that donated kidney three years ago, presented to the pain clinic with the severe pain around the left ear due to left side BP. The disease started acutely two weeks before, without any history of trauma or infectious disease. He examined by the neurologist in the emergency room of local hospital and outpatient neurologic clinic immediately after onset of the disease symptoms. The diagnosis of the BP was made according to history and physical examination. Other medical conditions like CVA, Lyme disease, viral infection, brain tumors were excluded. The patient was graduated as severe according to modified HouseBrackmann score. Prednisolone treatment started immediately, but due to side effects of corticosteroids and especially excessive blood pressure levels, the treatment was stopped. After explanations about pain clinic options for pain management in the clinic, the patient chose optional treatment by PST ${ }^{\circledR}$. The patient oral and written agreement were obtained. The standard protocol of nine days one-hour daily treatment of PST® started immediately on the home-based device of the PST ${ }^{\circledR}$. After sixth-day of the treatments recorded significant improvement in pain. Withdrawal of clinical signs of disease recorded at the eighth procedure. The patient completed nine days PST® treatment and fool recovery achieved within two months after acute onset of BP.

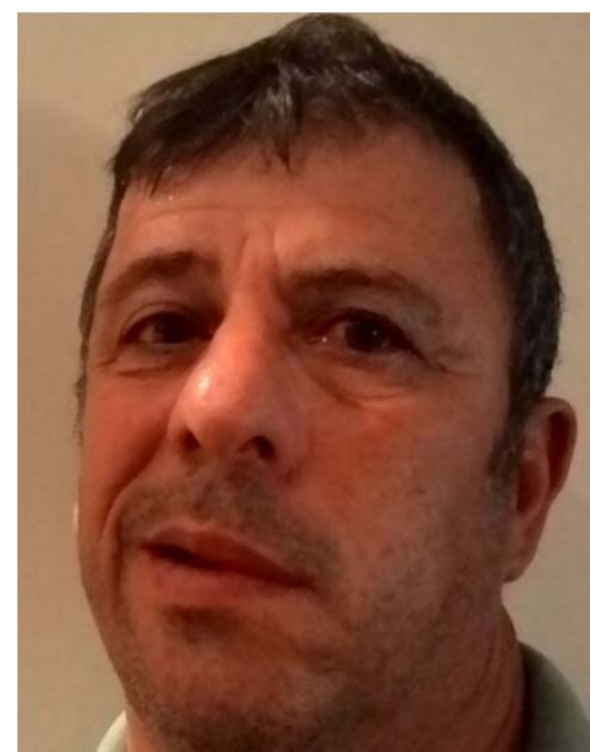

Figure 1: The clinical picture demonstrates the patient (case 2) two weeks after acute onset of BP and before started PST ${ }^{\circledR}$ treatment. 


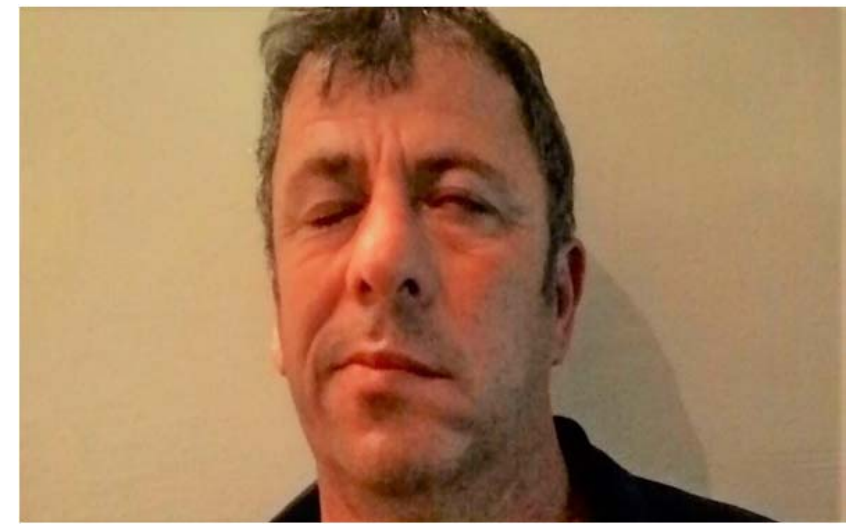

Figure 2: The clinical picture demonstrates the patient after sixth day of PST® treatment.

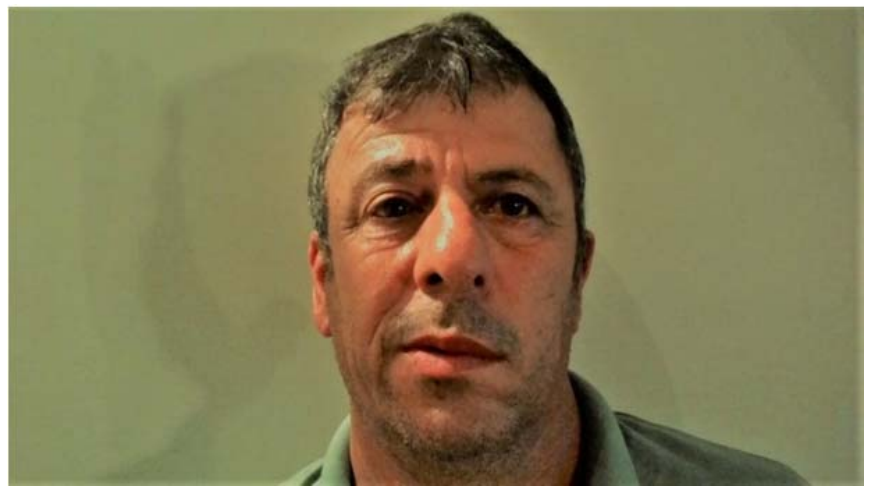

Figure 3: The clinical picture demonstrates the patient after the ninth day of PST® treatment.

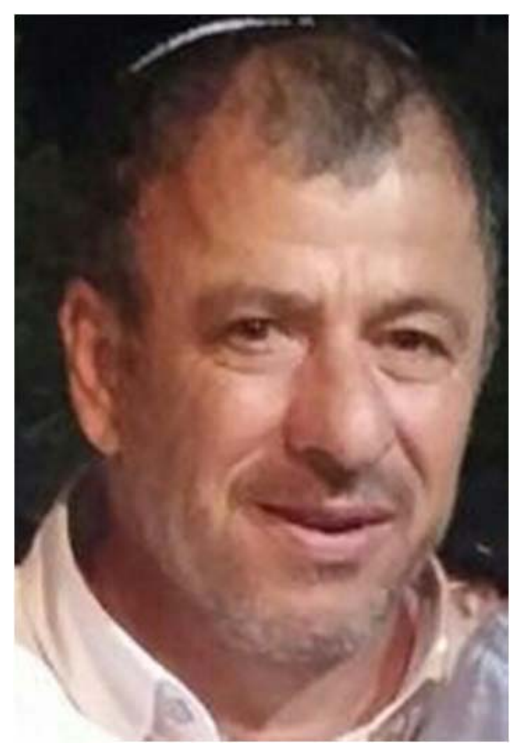

Figure 4: The clinical picture demonstrates the patient two months after PST ${ }^{\circledR}$ treatment. 


\section{Discussion}

BP is the facial paralysis that is not related to stroke and appeared as partial or complete paralysis. In the mild form of the disease full recovery recorded in most cases and without any complications even in patient that not received any therapy. However, in severe cases with complete facial muscle paralysis, 30\% have residual symptoms, and up to $12 \%$ have a recurrence [2]. Standard corticosteroid therapy of BP is recommended by guidelines in combined with other symptomatic treatments if needed [1]. However, diabetes, pregnancy, hypertension, and intolerance to corticosteroids may be contraindications to steroid therapy. No other medical modalities are known as useful for the treatment of BP.

In the reported cases authors represent patients that appeared in pain clinic with left side BP and complete paralysis of fascial muscles accompanied by severe ipsilateral pain around the ear. The patient was intolerant to steroid therapy and pain not responded to different kind of analgesics and NSAID. Patients treated by standard protocol of nine days one-hour daily treatment of PST ${ }^{\circledR}$ started immediately on the home-based device of the PST ${ }^{\circledR}$. In both cases, full recovery was recorded.

On the base of this cases, authors speculate that PST ${ }^{\circledR}$ may be effective in the treatment of different peripheral nerves diseases such as neuropathies, entrapment neuropathies, traumatic neuropraxia and diabetic peripheral neuropathy.

\section{Disclosure}

No author who contributed to this article has any conflict of interests to declare. All treatments and data collection performed in accordance with the laws of the State of Israel. The full clinical images presented during and after the treatment are published after receiving detailed written confirmation from the patient presented by the second case.

\section{References}

1. Baugh RF, Basura GJ, Ishii LE, et al. Clinical practice guideline: Bell's palsy. Otolaryngol Head Neck Surg 149 (2013): S1-S27.

2. Patel DK, Levin KH. Bell palsy: Clinical examination and management. Cleveland Clinic Journal of Medicine (CCJM) 82 (2015): 419-426.

3. D’Angelo C, Costantini E, Kamal MA, et al. Experimental model for ELF-EMF exposure: Concern for human health. Saudi J Biol Sci 22 (2015): 75-84.

4. Markov MS. Expanding use of pulsed electromagnetic field therapy. Electromagn Biol Med 26 (2007): 257-274.

5. Richard Markoll, et al. Pulsed Signal Therapy ${ }^{\circledR}$ for the treatment of musculoskeletal conditions: a millennium paradigm. APLAR Journal of Rheumatology 7 (2004): 292-305. 
Citation: Lauren Gorelick, Ayala Rozano-Gorelick. Full Recovery of the Patient With Bell's Palsy Within Two to Six Weeks After Single Course of Pulsed Electromagnetic Therapy-Case Reports. Fortune Journal of Health Sciences 1 (2018): 001-006.

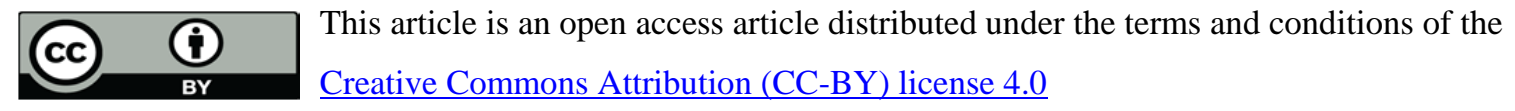

